Article

\title{
Compressed-Sensing-Based Time-Frequency Representation for Disturbance Characterization of Maglev On-Board Distribution Systems
}

\author{
Lu Xing ${ }^{1,2}$, Yinghong Wen ${ }^{1,2}$, Shi Xiao ${ }^{3}$, Jinbao Zhang ${ }^{1,2}$ and Dan Zhang ${ }^{1,2, *(\mathbb{C})}$ \\ 1 Electromagnetic Compatibility Laboratory, School of Electronic and Information Engineering, \\ Beijing Jiaotong University, Beijing 100044, China; 16111048@bjtu.edu.cn (L.X.); yhwen@bjtu.edu.cn (Y.W.); \\ jbzhang@bjtu.edu.cn (J.Z.) \\ 2 Beijing Engineering Research Center of EMC and GNSS Technology for Rail Transportation, Beijing 100044, China \\ 3 CRRC Qingdao Sifang Co., LTD., Qingdao 266111, China; xiaoshi@cqsf.com \\ * Correspondence: zhang.dan@bjtu.edu.cn
}

Received: 18 October 2020; Accepted: 11 November 2020; Published: 13 November 2020

check for updates

\begin{abstract}
The frequency variating source, linear generator, and switching devices lead to dynamic characteristics of the low-frequency conducted emissions within maglev on-board distribution systems. To track the time-varying feature of these disturbances, a joint time-frequency representation combined adaptive optimal kernel with compressed sensing technique is proposed in this paper. The joint representation is based on Wigner-Ville distribution, and employs adaptive optimal kernel to remove undesirable cross terms. The compressed sensing technique is introduced to deal with the tradeoff between cross-component reduction and auto-component smearing faced by kernel-function-based bilinear time-frequency representation. The time-frequency aggregation and accuracy performance of joint time-frequency representation is quantified using Rényi entropy and $l_{1}$-norm. To verify its performance in disturbance signature analysis for distribution systems and primarily characterize the low-frequency conducted emissions of maglev, a maglev on-board distribution system experimental platform is employed to extract the low-frequency disturbances which pose threats to the controlling system. Comparison with Wigner-Ville distribution demonstrates the joint time-frequency representation method outperforms in tracking time-varying and transient disturbances of maglev on-board distribution systems.
\end{abstract}

Keywords: electromagnetic disturbance; low-frequency noise; maglev train; conducted emission; time-frequency representation; distribution system

\section{Introduction}

The electromagnetic suspension (EMS) maglev is being rapidly developed as an efficient transportation tool featuring low noise levels, stable suspension and superior speed, the reliability and safety of an operating EMS maglev thus is being particularly considered worldwide [1-3]. As the maglev train is composed of various electrical and electronic devices, the electromagnetic emissions between the power supply system and the controlling system are unavoidable, which pose a threat to the stable operation of the maglev train $[4,5]$. As a result, the electromagnetic disturbance characterization and diagnosis form a crucial part of the manufacturing and security assessment procedures.

The major power supply within a maglev distribution system, the linear generator, exhibits time-varying harmonics characteristics as its output frequency is determined by the continuously changing velocity [3]. Moreover, common disturbance events in the distribution system, such as voltage sag, voltage swell, capacitor switching, etc. result in transient or short-duration 
waveforms [6-10]. As a result, time-frequency representation has drawn extensive attention as a better solution for disturbance characterization and diagnosis of various distribution systems [11-16]. The target signal would be distributed on the time-frequency plane as a 2-dimensional (2-D) pattern, showing different frequency components varying with time [17]. This feature makes the time-frequency analysis method a strong tool in extracting transient or instantaneous electromagnetic disturbance signals from background noise and identifying different signal components [18].

There are two main types of time-frequency representation in disturbance-characterizing applications, linear time-frequency representation and bilinear time-frequency representation. One universal linear time-frequency representation used in disturbance diagnosis is short-time Fourier transform (STFT) [19], which takes advantage of lower computational complexity and easy application. However, its time-frequency resolution is highly limited by the Heisenberg uncertainty, which leads to a tradeoff between its time localization performance and frequency localization performance [20]. Wigner-Ville distribution (WVD) is a bilinear time-frequency representation which describes the probability density function of signal energy simultaneously in the time and frequency domains [21]. The WVD can achieve high time-frequency resolution and has been successfully applied to various real-life signals [22-24]. However, for multicomponent signals, the WVD would produce cross term and interfere with the observation of signal time-frequency features [25]. In order to suppress the cross-term interference, numerous researchers are dedicated to developing WVD with new techniques. WVD admits an inner 2-D Fourier transform of ambiguity function (AF) [26]. In the ambiguity domain, the self terms of the target signal would spread along or around the axis, whereas the cross terms distribute away from the origin. Inspired by this feature, different types of low-pass filters, which are referred to as ambiguity-domain kernel functions, are proposed to suppress the cross terms in the ambiguity domain. Several classic kernel functions have shown superior performance in analyzing certain types of signal, e.g., Choi-Williams kernel [27], Rihaczek kernel [28], and Cone-shaped kernel [29]. In [30], Baraniuk and Jones designed a time-dependent radially-Gaussian-function-based kernel to adapt different types of time-varying signals. This adaptive optimal kernel shows superior performance in cross-term suppression and is applied to analyzing composite and real-life signal in various areas. As all the kernel-function-based bilinear time-frequency distributions require dealing with the tradeoff between cross-component reduction and auto-component smearing [20,26], we expand upon the preliminary results by introducing the Compressed Sensing Theory to find an optimal solution, and precisely characterize the time-frequency signature of low-frequency electromagnetic disturbance within maglev on-board distribution systems.

We organize the paper as follows. In Section 2, we firstly provide an illustration of the adaptive optimal kernel which we use to suppress the cross-term interference during bilinear time-frequency representation computing. Then, the steps to introduce Compressed Sensing Theory into the time-frequency representation are explained. In the end of this section, a composite signal is built to compare the performance between WVD and our joint mothed. Two important parameters, Rényi entropy and $l_{1}$-norm distance, are used to quantify the aggregation performance and accuracy of the two representations. Next, in Section 3, as the power supply circuit causes electromagnetic interference faults in the controlling units of maglev on-board distribution systems, an experimental platform is employed to capture the low-frequency noise within a maglev on-board distribution system. The time-frequency signature of measured disturbances is then described in detail according to joint time-frequency (TF) representation. Finally, Section 4 is our discussion.

\section{Methodology}

For a time domain signal, its Wigner-Ville Distribution (WVD) is described as:

$$
W V D(t, \omega)=\frac{1}{4 \pi^{2}} \iint A(\theta, \tau) e^{-j \theta t} e^{-j \tau \omega} d \theta d \tau
$$


where $A(\theta, \tau)$ refers to ambiguity function (AF) and is given by:

$$
A(\theta, \tau)=\int s\left(t+\frac{\tau}{2}\right) s^{*}\left(t-\frac{\tau}{2}\right) e^{j \theta t} d t
$$

where $s(t)$ is the analytic representation of target signal derived by Hilbert transform. It could be noticed from (1) that the ambiguity function could be regarded as an inner product between the target signal and its WVD, and each sample in ambiguity domain contains the time and frequency information of the signal. As a result, it is possible to build the time-frequency representation of the target signal by selecting the ambiguity-domain information.

When analyzing a composite signal $s(t)=s_{1}(t)+s_{2}(t)$, we write its WVD as:

$$
W V D_{s}(t, \omega)=W V D_{s_{1}}(t, \omega)+W V D_{s_{2}}(t, \omega)+2 \operatorname{Re}\left[W V D_{s_{1} s_{2}}(t, \omega)\right]
$$

where $W V D_{s_{1} s_{2}}$ is the cross Wigner-Ville distribution of the two signals and is given by:

$$
W V D_{s_{1} s_{2}}(t, \omega)=\int_{-\infty}^{+\infty} s_{1}\left(t+\frac{\tau}{2}\right) s_{2}{ }^{*}\left(t-\frac{\tau}{2}\right) e^{-j 2 \pi f \tau} d \tau
$$

The cross term creates non-zero values at unexpected locations between real signal trajectories in $(t, \omega)$ plane and, accordingly, is distributed away from the origin in $(\theta, \tau)$ plane. Driven by this feature, the cross term can be partially removed in ambiguity domain by a low-pass filter, which is called a kernel in bilinear time-frequency representation.

\subsection{Adaptive Optimal-Kernel}

The concept of Jones and Baraniuk's adaptive optimal kernel (AOK) is to build an adaptive signal-dependent kernel for each individual ambiguity-domain slice, and thus achieve better time-frequency localization performance in characterizing time-varying signals. To create the ambiguity-domain slices, a symmetrical window function $w(u)$ is defined as its value equal to zero for $|u|$ is larger than the preferred window size $T$. Then the short-time ambiguity function (STAF) indicating the ambiguity function of a relatively short interval of time surrounding the currently center time $t$ could be described as:

$$
\begin{aligned}
A(t ; \theta, \tau) & =\int R(u, \tau) w^{*}\left(u-t-\frac{\tau}{2}\right) w\left(u-t+\frac{\tau}{2}\right) e^{j \theta u} d u \\
& =\int s^{*}\left(u-\frac{\tau}{2}\right) w^{*}\left(u-t-\frac{\tau}{2}\right) s\left(u+\frac{\tau}{2}\right) w\left(u-t+\frac{\tau}{2}\right) e^{j \theta u} d u
\end{aligned}
$$

As Figure 1 shows, the symmetrical window $w(u)$ forms a rhombus-shaped area centered at $u=t_{0}$ in the domain correlating to the signal instantaneous signature. Only the signal component within the windowed area would be transformed into ambiguity domain and processed with kernel function.

Jones and Baraniuk designed a signal-dependent kernel based on radial Gaussian function to adapt the ambiguity-domain signal for a better cross-component suppression performance. The kernel is defined as:

$$
K_{\mathrm{opt}}(\theta, \tau)=\exp \left(-\frac{\theta^{2}+\tau^{2}}{2 \sigma^{2}(\psi)}\right)
$$

where $\sigma^{2}(\psi)$ refers to the spread function which controls the spread of the Gaussian at radial angle $\psi$. This kernel is adapted to a given signal in ambiguity domain by adjusting its scope and volume with the solution of an optimization problem. To better express the kernel feature, the optimization problem is defined in polar coordinate as:

$$
\max _{K_{\mathrm{opt}}} \int_{0}^{2 \pi} \int_{0}^{\infty}\left|A(r, \psi) K_{\mathrm{opt}}(r, \psi)\right|^{2} r d r d \psi
$$


subject to

$$
\begin{gathered}
K_{\mathrm{opt}}(r, \psi)=\exp \left(-\frac{r^{2}}{2 \sigma^{2}(\psi)}\right) \\
\frac{1}{4 \pi^{2}} \int_{0}^{2 \pi} \int_{0}^{\infty}\left|K_{\mathrm{opt}}(r, \psi)\right|^{2} r d r d \psi=\frac{1}{4 \pi^{2}} \int_{0}^{2 \pi} \sigma^{2}(\psi) d \psi \leq v, v \geq 0
\end{gathered}
$$

where $A(r, \psi)$ and $K_{\mathrm{opt}}(r, \psi)$ are the polar-coordinate expression of ambiguation function and optimal kernel function, and $r=\sqrt{\theta^{2}+\tau^{2}}$ refers to the radius variable in $(r, \psi)$ plane. Equation (8) constrains the kernel as a radially Gaussian kernel with lowpass property in the ambiguity domain, reserving signal's auto-components. Constraint (9) limits the volume of kernel to $v$. A large $v$ may let pass extra cross-components, whereas a small $v$ would smear the auto-component information, which raises a tradeoff between the cross-term interference suppression and localization performance in the time-frequency domain. To deal with this tradeoff, Jones and Baraniuk recommend choosing $v$ in the interval $[1,5]$ based on their study.

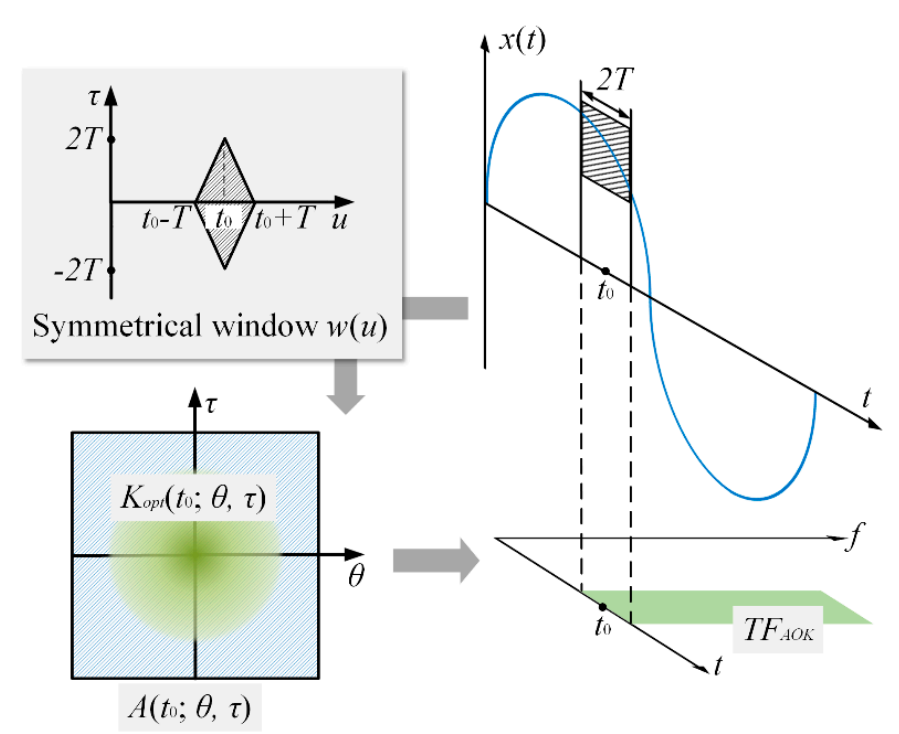

Figure 1. Principle of adaptive optimal-kernel time-frequency representation.

With the optimal kernel $K_{\text {opt }}$ determined by (7), an instantaneous slice of the windowed time-frequency representation could be computed as one slice of the 2-D Fourier transform of the STAF-kernel product at current time $t$ :

$$
T F_{A \mathrm{OK}}(t, \omega)=\frac{1}{4 \pi^{2}} \iint A(t ; \theta, \tau) K_{\mathrm{opt}}(t ; \theta, \tau) e^{-j \theta t} e^{-j \tau \omega} d \theta d \tau
$$

\subsection{Joint Time-Frequency Representation Based on Compressed Sensing}

For discrete-time data, the optimal kernel in the ambiguity domain could be regarded as a sampling area for time-frequency signal construction. To balance the tradeoff between cross-component reduction and auto-component smearing, various improved techniques are proposed to select a suitable collection of ambiguity-domain samples neighboring the origin. In this paper, we take advantage of the sparse sampling signature of Compressed Sensing Theory to find an optimal solution.

The basic concept of Compressed Sensing is to use far fewer samples than required by the Nyquist-Shannon Sampling Theory to reconstruct a signal with accuracy, which is numerically a linear inverse problem aiming to recover a high-dimensional signal from few linear measurements. 
A compressed sensing procedure of recovering a signal $x \in \boldsymbol{R}^{\mathrm{N}}$ from few measured samples $y \in \boldsymbol{R}^{\mathrm{M}}$ $(\mathrm{M}<<\mathrm{N})$ could be described as:

$$
y=\Phi x=\Phi \Psi z=A^{\mathrm{CS}} z
$$

where $\Phi$ refers to a $\mathrm{M} \times \mathrm{N}$ measurement matrix, z refers to the transformed-domain signal y processed by transformation matrix $\Psi$. $\Phi$ and $\Psi$ formed the so-called sensing matrix $A^{\mathrm{CS}}$, which is the crucial part in a compressed sensing system. Once the sensing matrix is determined, the high-dimension signal $y$ could be recovered by solving the following linear equation:

$$
\min _{z}\|z\|_{1} \text { s.t. } y=A^{C S} z
$$

where $\|z\|_{0}$ and $\|z\|_{1}$ refer to the $l_{0}$-norm and the $l_{1}$-norm of $z$, respectively.

By introducing Compressed Sensing Theory to the AOKTF, the tradeoff problem mentioned in Subsection A could be solved. We chose a sufficiently sampling area with AOK around the origin of the ambiguity plane and built a compressed sensing system to compute the signal's time-frequency distribution from the few ambiguity-domain sampling data. The restriction on parameter $v$ well removes the cross-component, whereas the compressed sensing process enables us to reconstruct the auto-term information from limited ambiguity-domain data.

The implementation of signal recovery by applying the compressed sensing technique is subject to two conditions, which are sparsity and incoherence constraints, respectively. The sparsity constraint indicates that the signal should not have much information in a certain transformed domain. The incoherence constraint indicates that the measurement matrix $\Phi$ and transformation matrix $\Psi$ must be incoherent. Detailed illustration of Compressed Sensing Theory and its constraints could refer to [31].

In the time-frequency domain, the signal energy is always distributed along the instantaneous frequency trajectory of each signal component. For a discrete-time signal with $M$ samples in the time domain, its time-frequency representation is of dimension $M^{2}$ when computed over $M$ frequency bins. The electromagnetic disturbances within the distribution system, such as harmonically-related disturbances, contain a limited number of frequency components. Thus assuming the quantity of signal components is $K$; where $K<<N$, there are at most $K \times N$ nonzero values on a targeted time-frequency spectrum. For wideband pulse signals within the distribution system, such as arc fault emission and transient emission caused by fast current $(d I / d t)$ and voltage $(d V / d t)$ transitions, the short duration guarantees the that signals are distributed across the time-frequency plane in a very sparse way. As a result, it is feasible to apply a compressed sensing technique in computing time-frequency representation to electromagnetic disturbance signature analysis.

To build a compressed sensing system for time-frequency representation, we describe the discrete-time-frequency representation windowed by AOK with a 2-D Fourier transform matrix $F_{2 \mathrm{D}}$ as:

$$
\mathbf{T F}_{A \mathrm{OK}}=\boldsymbol{F}_{2 \mathrm{D}}\left\{\boldsymbol{T F} F_{A \mathrm{OK}}\right\} \cdot A \cdot K_{\mathrm{opt}}
$$

For each short-time ambiguity-domain slice, we regard the windowed ambiguity-domain signal $A \cdot K_{\text {opt }}$ as the measurements y in the compressed sensing system defined in (10), then the compressed sensing representation for solving target time-frequency distribution could be described as:

$$
A \cdot K_{\mathrm{opt}}=\boldsymbol{F}_{2 \mathrm{D}}^{-1}\left\{\boldsymbol{T F _ { A O K } \}} \cdot \mathbf{T F _ { A O K }}\right.
$$

where $F_{2 \mathrm{D}}^{-1}$ is the inverse 2-D Fourier transform matrix and is regarded as the compressed sensing matrix like $A^{\mathrm{CS}}$ in (11). As a result, the target time-frequency distribution $\mathbf{T F _ { \mathrm { AOK } }}$ is regarded as the high-dimension signal y to be recovered in this compressed sensing system. Then, according to 
compressed sensing theory, the target time-frequency representation could be obtained by solving the $l_{1}$-minimization problem:

$$
\min \left\|\boldsymbol{T F}_{A \mathrm{OK}}\right\|_{1} \text { s.t. }\left\|\boldsymbol{A} \cdot \boldsymbol{K}_{\mathrm{opt}}-\boldsymbol{F}_{2 \mathrm{D}}^{-1} \cdot \boldsymbol{T F}_{A \mathrm{OK}}\right\|_{2}=0
$$

\subsection{Performance of Joint TF Representation}

The time-frequency aggregation and accuracy in the time-frequency domain are two important properties in signal analysis. To evaluate the two properties of the proposed joint method, we compare the spectrogram under WVD and joint TF representation of a composite signal. The signal is composed of a linear frequency modulation (LFM) signal and a sinusoidal frequency modulation (SFM) signal:

$$
\begin{gathered}
y_{\mathrm{LFM}}(t)=\exp \left(j 2 \pi\left(f_{0} t+\frac{1}{2} \mu t^{2}\right)\right) \\
y_{\mathrm{SFM}}(t)=\exp \left(j 2 \pi f_{\mathrm{c}} t+j m_{f} \sin \left(2 \pi f_{\mathrm{m}} t\right)\right)
\end{gathered}
$$

The ideal time-frequency distribution of the two frequency modulation signals are:

$$
\begin{gathered}
f_{\mathrm{LFM}}(t)=f_{0}+\mu t \\
f_{\mathrm{SFM}}(t)=f_{\mathrm{c}}+m_{f} f_{\mathrm{m}} \cos \left(2 \pi f_{\mathrm{m}} t\right)
\end{gathered}
$$

where $f_{0}=100 \mathrm{~Hz}, \mu=200, f_{\mathrm{c}}=250 \mathrm{~Hz}, m_{f}=100, f_{\mathrm{m}}=2 \mathrm{~Hz}$ in this paper. The time-domain symmetrical window is 64 samples long for joint TF, whereas the volume $v$ of AOK is defined as 3 .

We built the time-frequency spectrogram of the composite signal with the real-part data as Figure 2 shows. It is two distinct trajectories on the spectrograms were expected. However, from Figure $2 \mathrm{a}$ it can be seen that the signal trajectories are covered by severe cross-term interference. The cross-term interference mostly happens at the turning and crossing parts of the signal tracks. The cross-terms form a quasi-frequency-modulated signal trajectory whose frequency sweeps from $100 \mathrm{~Hz}$ to $350 \mathrm{~Hz}$ on WVD representation and makes the signal indistinguishable. By contrast, as is shown in Figure $2 b$, the time-frequency spectrogram represented by joint TF provides a clear insight into the signal component; the cross-term interference is well under control.

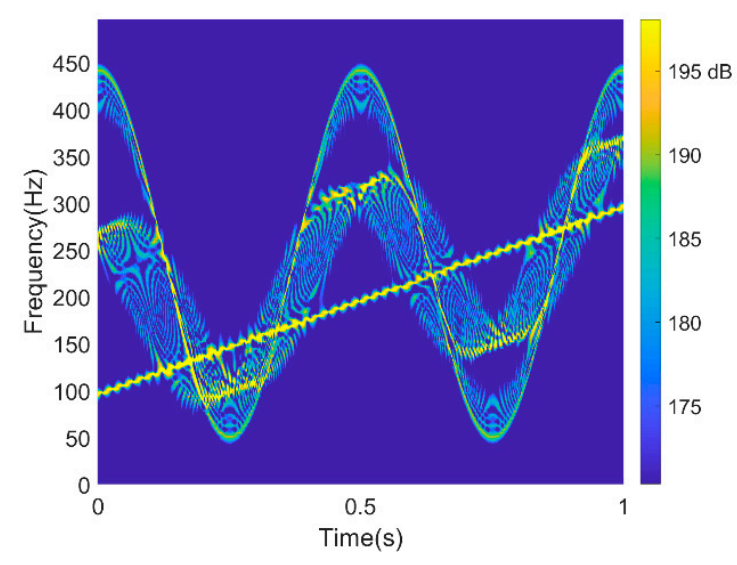

(a)

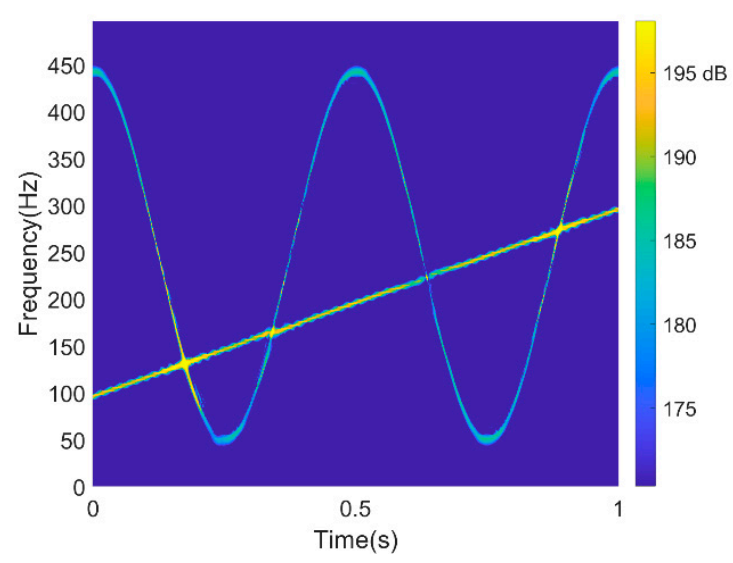

(b)

Figure 2. Time-frequency representations of the composite signal by (a) Wigner-Ville distribution (WVD) and (b) joint TF representation.

To quantify the time-frequency aggregation and accuracy of the two representations, we mapped the time-frequency representation data into normalized grayscale image data and then computed 
the Rényi entropy and $l_{1}$-norm between the computed spectrogram and the ideal spectrogram of the composite signal, respectively.

Rényi entropy is considered to be a measure of diversity, uncertainty or randomness of an information system. It has been introduced to quantify features in a time-frequency spectrogram [32]. A smaller Rényi entropy indicates a lower complexity of information, which refers to higher time-frequency aggregation for a time-frequency domain signal. Thus, the time-frequency aggregation performance of a certain representation method can be measured.

Defining the time-frequency representation computed by certain method as $X(t, \omega)$, then, its Rényi entropy of order $\alpha$, where $\alpha$ is normally set to 3 in time-frequency features extraction, is defined as:

$$
R_{\alpha}=\frac{1}{1-\alpha} \log \left(\sum_{t} \sum_{\omega} P^{\alpha}(t, \omega)\right)
$$

where $\boldsymbol{P}(t, \omega)$ refers to the probability distribution of $\boldsymbol{X}(t, \omega)$ with unit energy and is defined as:

$$
\boldsymbol{P}(t, \omega)=\frac{\boldsymbol{X}(t, \omega) \boldsymbol{X}^{*}(t, \omega)}{\sum_{t} \sum_{\omega}\left(\boldsymbol{X}(t, \omega) \boldsymbol{X}^{*}(t, \omega)\right)}
$$

The $l_{1}$-norm can be used to measure the deference between the ideal time-frequency spectrogram and the time-frequency spectrogram represented by a certain method, and thus the accuracy becomes comparable [33]. Defining the ideal time-frequency representation as $Y(t, \omega)$, then the $l_{1}$-norm distance between the ideal representation and the computed representation is:

$$
D_{l_{1}}=\sum_{t} \sum_{\omega}|\boldsymbol{Y}(t, \omega)-X(t, \omega)|
$$

A small value of $l_{1}$-norm distance indicates lower discrepancy between the computed spectrogram and the ideal model, and thus the accurate performance can be measured.

Using (20) and (22), we can measure the performance of the proposed joint time-frequency representation and WVD. The Rényi entropy and $l_{1}$-norm distance of the two representations are shown in Table 1. The Rényi entropy in Table 1 indicates slight variation of the aggregation performance between the compared distributions. Meanwhile, as a result of the effective cross-term suppression, the joint TF representation shows $72.85 \%$ reduction in $l_{1}$-norm distance compared to WVD, which is a significant improvement in accuracy. Taking the above benefits of joint TF, we can better extract the characteristics of time-varying disturbances within the maglev distribution system.

Table 1. Performance comparison.

\begin{tabular}{ccc}
\hline & Rényi Entropy & $\boldsymbol{l}_{\mathbf{1}}$-Norm \\
\hline Ideal Representation & 7.4761 & 0 \\
WVD & 9.4079 & 2525.4677 \\
Joint TF Representation & 8.0946 & 685.7371 \\
\hline
\end{tabular}

\section{Application}

The maglev distribution system experimental platform used in this paper is shown in Figure 3. This platform was constructed according to the actual design of the China high-speed maglev system and was built in a laboratory environment. The experimental platform is capable of carrying out power quality evaluation and conducted emission measurement for various loads and operating conditions of the maglev train.

The networking mode of the maglev on-board distribution system is described in Figure 4. The main circuit equipment is powered up by the DC $440 \mathrm{~V}$ network, whereas the DC $24 \mathrm{~V}$ network provides power to switching boxes and controlling equipment. All the on-board equipment is controlled by the on-board distribution system controlling unit, which sends controlling signals under 
the operating instruction from central controlling unit. To implement all the power supply conditions with the experimental platform, a simulated linear generator and a simulated power supply rail were designed to simulate the maglev external power sources. Imitating the real linear generator applied in the maglev system, the parameters of the simulated linear generator fluctuate with maglev velocity.

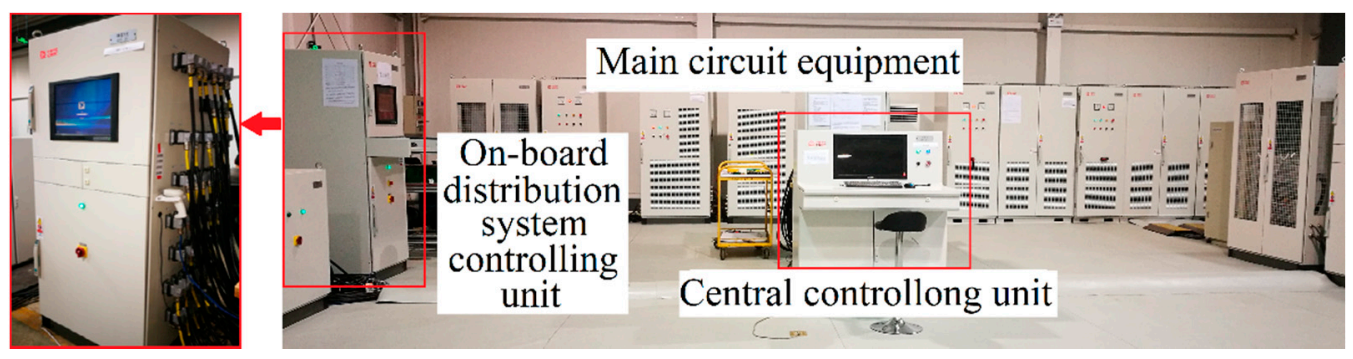

Figure 3. Maglev distribution system experimental platform.

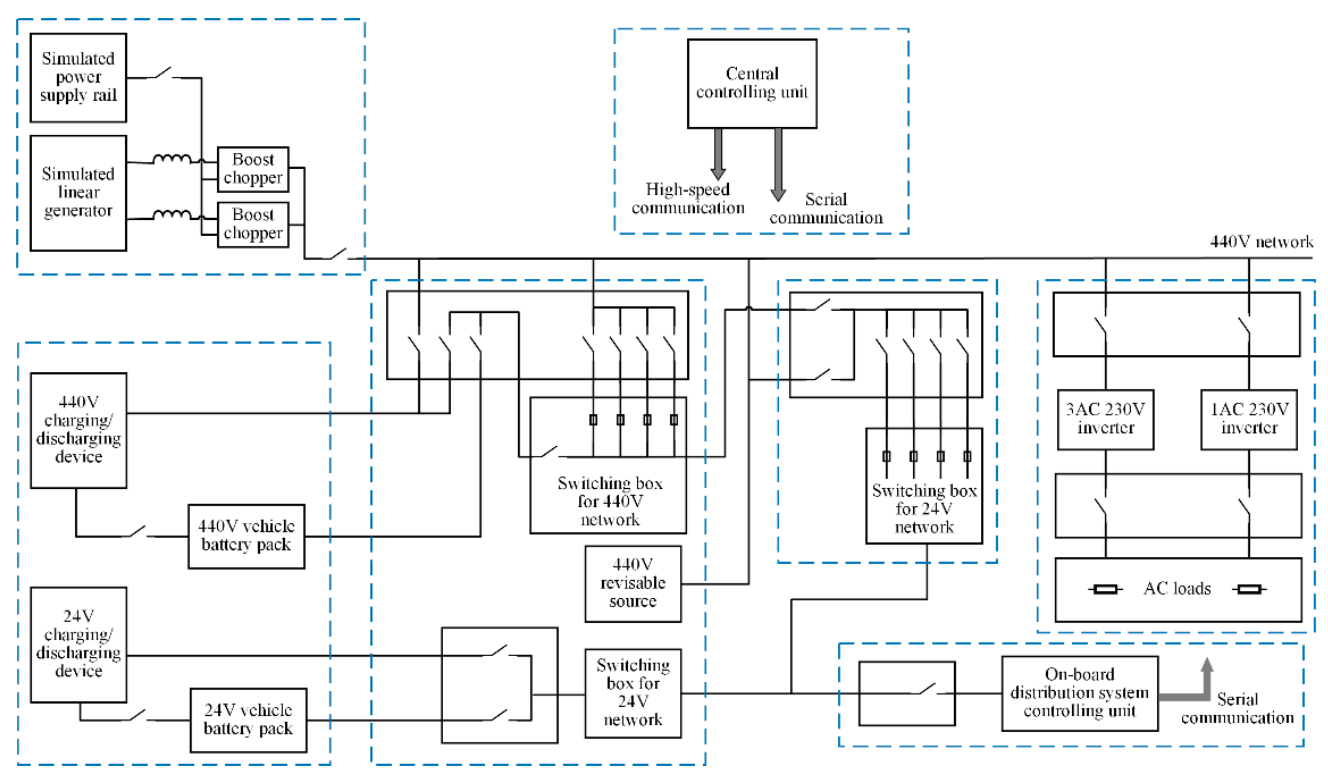

Figure 4. Networking mode of the maglev on-board distribution system.

As a complex low-voltage high-current distributed power supply system with redundancy, the maglev on-board distribution system has seen various electromagnetic interference (EMI) faults during its controlling and operating:

- The signal acquisition of controlling units during transition from the start-up stage to the accelerating stage is interfered, which makes the host computer of controlling units freeze. Experimental results demonstrate that common-mode disturbance on the controlling unit signal line appears during the transition and is directly related to the disturbance on linear generator output.

- A periodic fluctuation is captured on a battery charging cable of a $440 \mathrm{~V}$ on-board vehicle battery pack, which leads to an abnormal low-frequency radiation characteristic around the battery pack and pose threats to adjacent equipment.

As the in-depth cognition of disturbances characteristic is the precondition of an anti-EMI technique, we employed the mentioned experimental platform to monitor the disturbances within the maglev on-board distribution system, and used the joint time-frequency representation as an analysis tool in signature characterization. 


\subsection{Low-Frequency Disturbance Characteristic during Transition from the Start-Up Stage to the Accelerating Stage}

During this period, the linear generator transits from the static state to operating mode, with its output frequency increasing as the train accelerates. The measured time series and its frequency-domain component during this transition are shown as Figure 5a,b. The time-frequency representation of the normalized common-mode voltage, which is measured on the output of linear generator, is shown in Figure 6. The sample frequency of the measurement is $1 \mathrm{kHz}$.

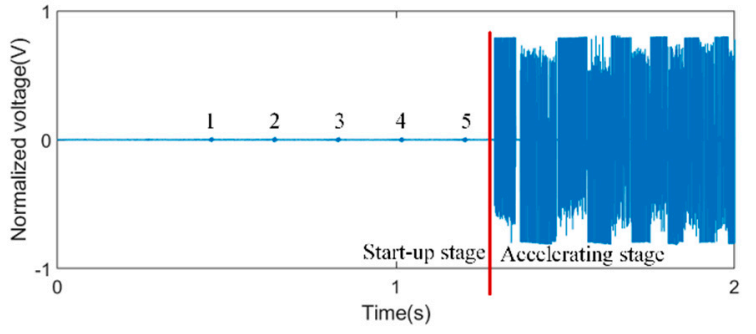

(a)

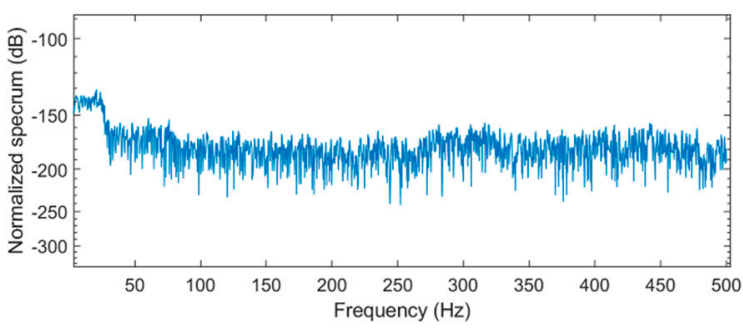

(b)

Figure 5. Common mode voltage of linear generation output in the (a) time domain and (b) frequency domain.

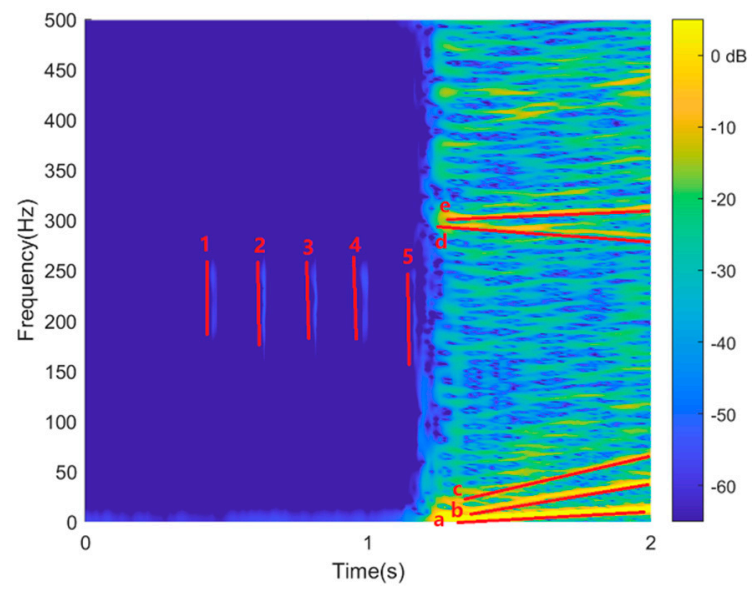

(a)

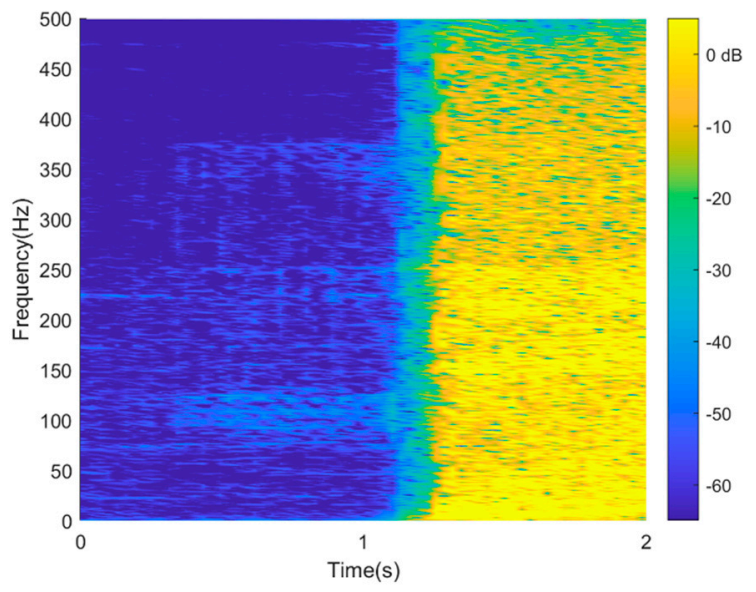

(b)

Figure 6. Time-frequency spectrogram of common-mode voltage of the linear generator represented by (a) joint TF and (b) WVD.

As is shown in Figure 5a, during the start-up stage, there are five slight but visible fluctuations within a cycle of $180 \mathrm{~ms}$ on the time-domain signal of the linear generator. The normalized spectrum of the output voltage demonstrates that the largest frequency component of the output is DC. Meanwhile, the disturbance energies in 0-30, 280-300 and 300-330 Hz frequency bands are slightly higher than in the other frequency bands.

According to Figure $6 \mathrm{a}$, the time-frequency spectrogram of the linear generator during this period is clearly divided into two parts before and after the transition. In start-up stage, the fluctuations on the linear generator output could be clearly addressed by our proposed joint time-frequency representation, showing its frequency components mainly distributed between $150 \mathrm{~Hz}$ and $250 \mathrm{~Hz}$.

An increase in noise level up to $50 \mathrm{~dB}$ after the transition could be observed according to Figure 6a. However, there are still five distinct trajectories on the accelerating stage spectrogram. Trajectory a is the ideal output voltage of the linear generator as it is the most concentrated energy trajectory on the spectrogram, whereas the other trajectories address the disturbance characteristics on the measured point. Showing a similar frequency-variation feature with Trajectory a, Trajectory b and Trajectory c are 
supposed to be low-order harmonics following the linear generator output. Trajectory $\mathrm{d}$ and Trajectory $\mathrm{e}$, however, are unexpected noise appearing at this stage, and are supposed to be the time-frequency reflection of disturbance components within the $280-300 \mathrm{~Hz}$ and $300-330 \mathrm{~Hz}$ frequency bands observed from Figure 5b.

The time-frequency spectrograms of the measured voltages represented by WVD are shown in Figure $6 \mathrm{~b}$ for comparison. The WVD shows its superior time-frequency aggregation performance according to Figure 6b. However, comparing with Figure 6a, WVD shows its drawback in displaying the time-frequency feature accurately. The fluctuations and trajectories with the frequency-variation feature of the linear generator output could not be discerned from Figure $6 \mathrm{~b}$, which is supposed to be a result of severe cross-term interference

\subsection{Low-Frequency Disturbance Characteristic on the Battery Charging Line}

During the stable operating mode, the overall electricity demand of the on-board electrical and electronic system fully depends on the linear generator. Under this condition, $440 \mathrm{~V}$ vehicle battery stops discharging and starts recharging.

An abnormal noise waveform is captured on the charging line for the $440 \mathrm{~V}$ vehicle battery pack after full charging. The time-domain waveform of the measured noise and its spectrum are shown in Figure 7. The sample frequency of the measurement is $10 \mathrm{kHz}$.

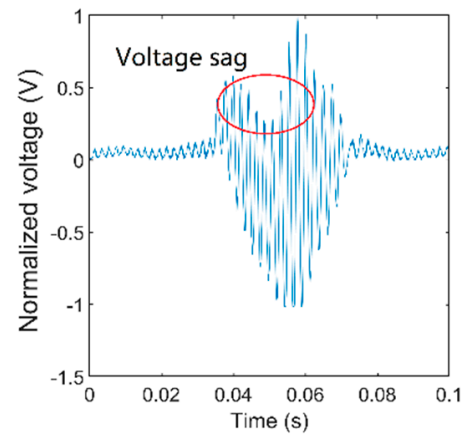

(a)

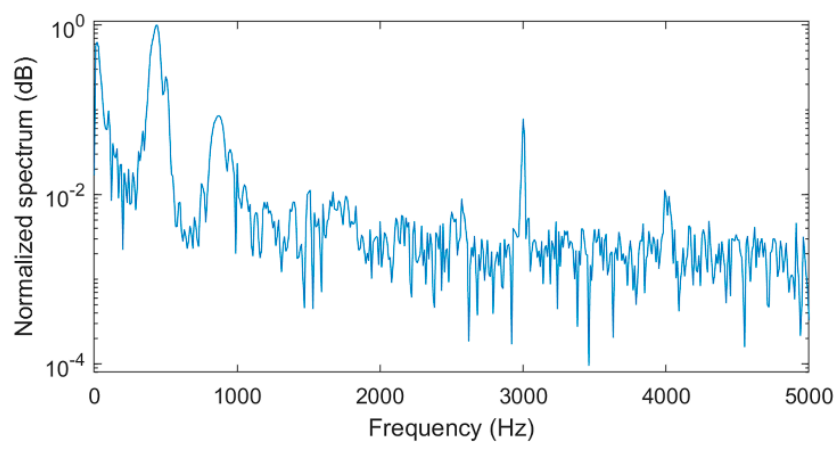

(b)

Figure 7. Common-mode disturbance on the $440 \mathrm{~V}$ battery pack charging cable after full charging in the (a) time domain and (b) frequency domain.

The disturbance appears with a cycle of about $0.1 \mathrm{~s}$. From Figure $7 \mathrm{a}$ it could be noticed that the single cycle disturbance forms a heart-shaped waveform in the time domain with a voltage sag at the middle of the disturbance waveform. The spectrum of the single cycle disturbance shows that the signal energy mainly distributes within four frequency bands: DC-50, 350-550, 800-1000 and 2950-3050 Hz.

The time-frequency spectrogram of single cycle disturbance is represented by joint TF as Figure 8a. The TF spectrogram addresses the wide-band transient feature at the beginning of the voltage sag. Three frequency components could be distinguished from the 3-D view of the joint TF representation: the DC component, $400 \mathrm{~Hz}$ component and $500 \mathrm{~Hz}$ component. The $500 \mathrm{~Hz}$ component appears along with the wide-band transient, whereas the $400 \mathrm{~Hz}$ component appears at the middle of the disturbance time series. However, the $800-1000 \mathrm{~Hz}$ and $2950-3050 \mathrm{~Hz}$ frequency components are not clearly addressed according to joint TF.

As is shown in Figure 9, by adjusting the color bar scale and focusing on the disturbance other than the heart-shaped waveform part, we can dimly notice that there are few constant-frequency trajectories running through the time-frequency spectrogram. The trajectories around $1000 \mathrm{~Hz}$ and $3000 \mathrm{~Hz}$ are supposed to correspond to the $800-1000 \mathrm{~Hz}$ and $2950-3050 \mathrm{~Hz}$ frequency components 
in the frequency domain. The trajectory around $1000 \mathrm{~Hz}$ is less obvious and almost covered by the background noise.

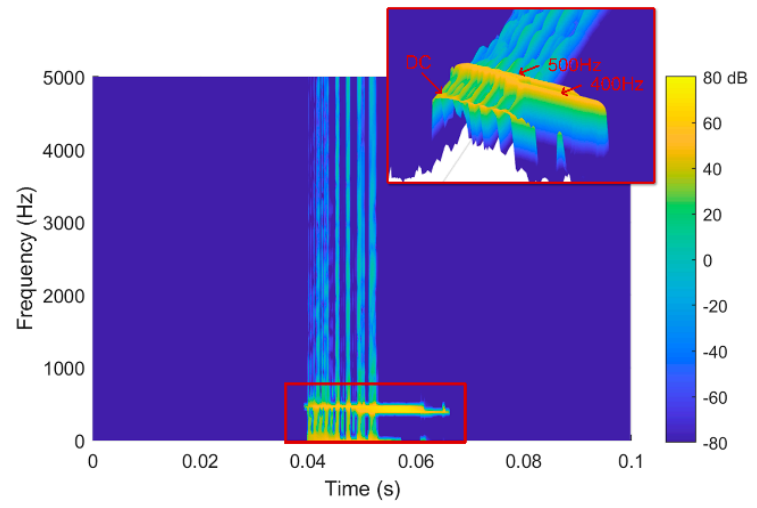

(a)

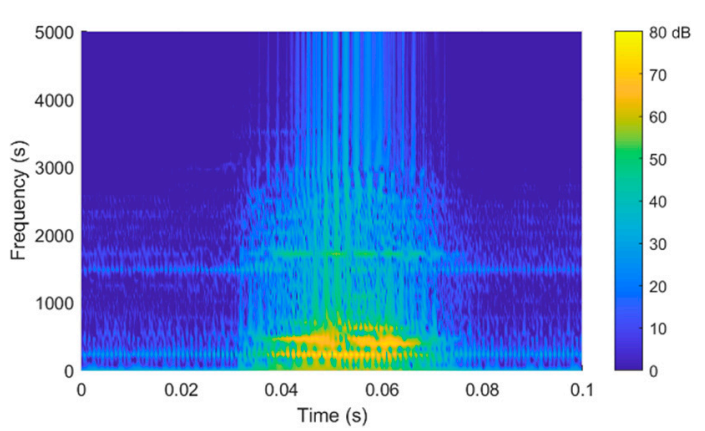

(b)

Figure 8. Time-frequency spectrogram of the measured common-mode disturbance on the battery charging cable represented by (a) joint TF and (b) WVD.

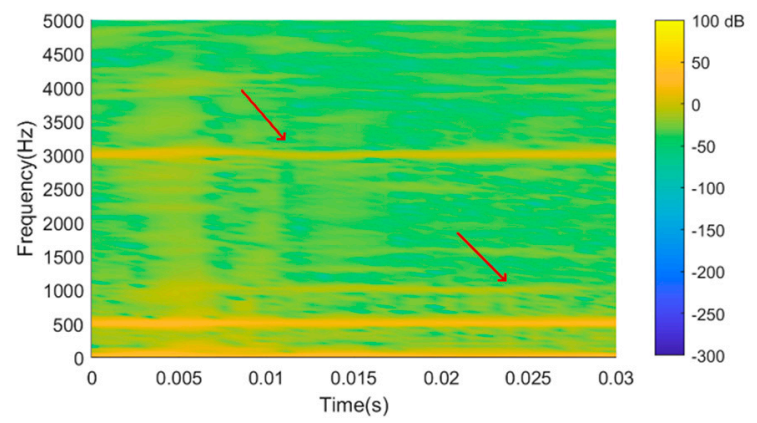

Figure 9. A zooming-in of the time-frequency spectrogram in Figure 8a.

The time-frequency spectrogram of measured disturbance represented by WVD is shown as Figure $8 \mathrm{~b}$. The WVD spectrogram shows that the produced cross term interference forms a strong trajectory around $200 \mathrm{~Hz}$ between the actual signal time-frequency components, which makes it difficult to determine the actual time-frequency characteristic of disturbance. The $800-1000 \mathrm{~Hz}$ and 2950-3050 Hz frequency components are not clearly addressed by WVD either.

\section{Discussion}

In Section 3, it was shown that the joint TF representation works well in capturing the frequency variation feature of electromagnetic disturbance, transient disturbance and could also be well addressed by the joint method. However, in the case of analyzing the disturbance signature on the vehicle battery charging line, the joint TF method did not provide satisfying insight into the continuous disturbance with constant frequency.

As the time-frequency representation describes the signal energy distribution simultaneously in the time-frequency domain, it could highlight that the high-energy-density components in the time-frequency spectrogram, however, smears the evenly-distributed components at the same time. Subject to this drawback of time-frequency representation, it is important to choose a suitable analysis scope of the time series, avoiding distributing high-energy transient disturbance and continuous stationary disturbance on the same time-frequency spectrogram view. As harmonics and inter-harmonics are common components of maglev disturbance, time-frequency representation shows obvious drawbacks in distinguishing this type of disturbance from voltage sag, capacitor switching 
or arc-fault disturbance. The continuous stationary signal could be addressed by adjusting the view scope and color bar, but still easily be overlooked without a previous insight into the signal component. Meanwhile, the optimization procedures during optimal kernel searching and compressed-sensing-based time-frequency spectrogram building lead to heavier computation than WVD and the linear time-frequency representation method. Thus it is better to use the time-domain and frequency-domain signature as a reference to choose a suitable view scope for time-frequency characterization. Overall, the proposed time-frequency shows better performance than WVD and is proven to be a useful tool in time-frequency characterization of disturbance within maglev on-board distribution systems.

\section{Conclusions}

In this paper, a joint time-frequency representation is proposed to precisely address the time-varying low-frequency disturbance signature within maglev on-board distribution systems. To optimize the time-frequency aggregation performance and accuracy of the time-frequency representation synchronously, we employ an adaptive optimal kernel to suppress the cross-term interference and introduced a compressed sensing technique to recover self-term information. The third-order Rényi entropy of joint TF representation and $l_{1}$-norm to the ideal model confirm the outperformance of joint TF. The joint TF representation was further applied to analyzing and characterizing conducted emissions measured from a maglev on-board distribution system experimental platform. Spectrograms of the measured result show that the joint TF representation works well in catching the frequency variation feature of electromagnetic disturbance; transient and short-duration disturbance could also be well addressed by the joint method. As a result, the proposed joint TF could be used as a useful tool in electromagnetic disturbance characterization and power quality evaluation of maglev systems.

Author Contributions: Conceptualization, L.X. and Y.W.; methodology, L.X. and D.Z.; software, L.X.; validation, Y.W., S.X. and J.Z.; writing—original draft preparation, L.X. and D.Z.; writing—review and editing, L.X. and J.Z.; supervision, Y.W.; project administration, S.X.; funding acquisition, Y.W. All authors have read and agreed to the published version of the manuscript.

Funding: This work was funded by National Natural Science Foundation of China (U1734203) and Fundamental Research Funds for the Central Universities (2018YJS020).

Conflicts of Interest: The authors declare no conflict of interest.

\section{References}

1. Glatzel, K.; Khurdok, G.; Rogg, D. The development of the magnetically suspended transportation system in the federal Republic of Germany. IEEE Trans. Veh. Technol. 1980, 29, 3-17. [CrossRef]

2. Park, D.Y.; Shin, B.C.; Han, H. Korea's Urban Maglev Program. Proc. IEEE 2009, 97, 1886-1891. [CrossRef]

3. Morishita, M.; Azukizawa, T.; Kanda, S.; Tamura, N.; Yokoyama, T. A new MAGLEV system for magnetically levitated carrier system. IEEE Trans. Veh. Technol. 1989, 38, 230-236. [CrossRef]

4. Lee, H.-W.; Kim, K.-C.; Lee, J. Review of maglev train technologies. IEEE Trans. Magn. 2006, 42, 1917-1925.

5. Boldea, I.; Trica, A.; Papusoiu, G.; Nasar, S.A. Field tests on a MAGLEV with passive guideway linear inductor motor transportation system. IEEE Trans. Veh. Technol. 1988, 37, 213-219. [CrossRef]

6. Islam, M.; Mohammadpour, H.A.; Ghaderi, A.; Brice, C.W.; Shin, Y. Time-Frequency-Based Instantaneous Power Components for Transient Disturbances According to IEEE Standard. IEEE Trans. Power Deliv. 2015, 30, 1288-1297. [CrossRef]

7. Chen, H.; Qian, Z. Modeling and Characterization of Parasitic Inductive Coupling Effects on Differential-Mode EMI Performance of a Boost Converter. IEEE Trans. Electromagn. Compat. 2011, 53, 1072-1080. [CrossRef]

8. Lai, J.-S.; Huang, X.; Chen, S.; Nehl, T.W. EMI characterization and simulation with parasitic models for a low-voltage high-current AC motor drive. IEEE Trans. Ind. Appl. 2004, 40, 178-185. [CrossRef] 
9. Sun, A.G.; Crofton, M.W.; Young, J.A.; Cox, W.A.; Beiting, E.J. L-, S-, and C-Band EMI Measurement and Characterization of Spacecraft ESD Events. IEEE Trans. Plasma Sci. 2013, 41, 3505-3511. [CrossRef]

10. Azpúrua, M.A.; Pous, M.; Silva, F. Decomposition of Electromagnetic Interferences in the Time-Domain. IEEE Trans. Electromagn. Compat. 2016, 58, 385-392. [CrossRef]

11. Wang, H.; Liu, J.; Luo, S.; Xu, X. Research on Power Quality Disturbance Detection Method Based on Improved Ensemble Empirical Mode Decomposition. Electronics 2020, 9, 585. [CrossRef]

12. Das, S.R.; Ray, P.K.; Sahoo, A.K.; Ramasubbareddy, S.; Babu, T.S.; Kumar, N.M.; Haes Alhelou, H.; Siano, P. Performance of Hybrid Filter in a Microgrid Integrated Power System Network Using Wavelet Techniques. Appl. Sci. 2020, 10, 6792. [CrossRef]

13. Marnerides, A.K.; Smith, P.; Schaeffer-Filho, A.; Mauthe, A. Power Consumption Profiling Using Energy Time-Frequency Distributions in Smart Grids. IEEE Commun. Lett. 2015, 19, 46-49. [CrossRef]

14. Yang, R.; Zhang, B.; Qiu, D.; Liu, Z. Time-Frequency and Wavelet Transforms of EMI Dynamic Spectrum in Chaotic Converter. IEEE Trans. Power Electron. 2009, 24, 1083-1092. [CrossRef]

15. Cho, W.; Powers, E.J. Time-frequency analysis for the grid power quality of a variable speed wind turbine. In Proceedings of the 2009 IEEE Power Electronics and Machines in Wind Applications, Lincoln, NE, USA, 24-26 June 2009; pp. 1-6.

16. Gu, Y.H.; Bollen, M.H.J. Time-frequency and time-scale domain analysis of voltage disturbances. IEEE Trans. Power Deliv. 2000, 15, 1279-1284. [CrossRef]

17. Cho, S.; Jang, G.; Kwon, S. Time-Frequency Analysis of Power-Quality Disturbances via the Gabor-Wigner Transform. IEEE Trans. Power Deliv. 2010, 25, 494-499.

18. Sezgin, E.; Salor, Ö. Analysis of Power System Harmonic Subgroups of the Electric Arc Furnace Currents Based on a Hybrid Time-Frequency Analysis Method. IEEE Trans. Ind. Appl. 2019, 55, 4398-4406. [CrossRef]

19. Garcia, C.I.; Grasso, F.; Luchetta, A.; Piccirilli, M.C.; Paolucci, L.; Talluri, G. A Comparison of Power Quality Disturbance Detection and Classification Methods Using CNN, LSTM and CNN-LSTM. Appl. Sci. 2020, 10, 6755. [CrossRef]

20. Whitelonis, N.; Ling, H. Radar Signature Analysis Using a Joint Time-Frequency Distribution Based on Compressed Sensing. IEEE Trans. Antennas Propag. 2014, 62, 755-763. [CrossRef]

21. Abeysekera, R.; Bolton, R.; Westphal, L.; Boashash, B. Patterns in Hilbert transforms and Wigner-Ville distributions of electrocardiogram data. In Proceedings of the ICASSP '86. IEEE International Conference on Acoustics, Speech, and Signal Processing, Tokyo, Japan, 7-11 April 1986; Volume 11, pp. 1793-1796.

22. Boashash, B.; White, L.; Imberger, J. Wigner-Ville analysis of non-stationary random signals. (with application to turbulent microstructure signals). In Proceedings of the ICASSP '86. IEEE International Conference on Acoustics, Speech, and Signal Processing, Tokyo, Japan, 7-11 April 1986; Volume 11, pp. 2323-2326.

23. Huang, P.; Liao, G.; Yang, Z.; Xia, X.; Ma, J.; Zhang, X. A Fast SAR Imaging Method for Ground Moving Target Using a Second-Order WVD Transform. IEEE Trans. Geosci. Remote Sens. 2016, 54, 1940-1956. [CrossRef]

24. Cai, K.; Cao, W.; Aarniovuori, L.; Pang, H.; Lin, Y.; Li, G. Classification of Power Quality Disturbances Using 3Wigner-Ville Distribution and Deep Convolutional Neural Networks. IEEE Access 2019, 7, 119099-119109. [CrossRef]

25. Flandrin, P. Some features of time-frequency representations of multicomponent signals. In Proceedings of the ICASSP '84. IEEE International Conference on Acoustics, Speech, and Signal Processing, San Diego, CA, USA, 19-21 March 1984; Volume 9, pp. 266-269.

26. Flandrin, P.; Borgnat, P. Time-Frequency Energy Distributions Meet Compressed Sensing. IEEE Trans. Signal Process. 2010, 58, 2974-2982. [CrossRef]

27. Barry, D.T. Fast calculation of the Choi-Williams time-frequency distribution. IEEE Trans. Signal Process. 1992, 40, 450-455. [CrossRef]

28. Scharf, L.L.; Schreier, P.J.; Hanssen, A. The Hilbert space geometry of the Rihaczek distribution for stochastic analytic signals. IEEE Signal Process. Lett. 2005, 12, 297-300. [CrossRef]

29. Zhao, Y.; Atlas, L.E.; Marks, R.J. The use of cone-shaped kernels for generalized time-frequency representations of nonstationary signals. IEEE Trans. Acoust. Speech Signal Process. 1990, 38, 1084-1091. [CrossRef]

30. Jones, D.L.; Baraniuk, R.G. An adaptive optimal-kernel time-frequency representation. IEEE Trans. Signal Process. 1995, 43, 2361-2371. [CrossRef]

31. Donoho, D.L. Compressed sensing. IEEE Trans. Inf. Theory 2006, 52, 1289-1306. [CrossRef] 
32. Baraniuk, R.G.; Flandrin, P.; Michel, O. Measuring time-frequency information and complexity using the Renyi entropies. IEEE Trans. Inf. Theory 2001, 47, 1391-1409. [CrossRef]

33. Michel, O.; Baraniuk, R.G.; Flandrin, P. Time-frequency based distance and divergence measures. In Proceedings of the IEEE-SP International Symposium on Time-Frequency and Time-Scale Analysis, Philadelphia, PA, USA, 6 August 2002.

Publisher's Note: MDPI stays neutral with regard to jurisdictional claims in published maps and institutional affiliations.

(C) 2020 by the authors. Licensee MDPI, Basel, Switzerland. This article is an open access article distributed under the terms and conditions of the Creative Commons Attribution (CC BY) license (http://creativecommons.org/licenses/by/4.0/). 